raten mit starker Kompensation ist die Möglichkeit gegeben, daß sich auch im Innern des Materials, z. B. längs Versetzungslinien Inversions,,bänder“ ausbilden.

Auch bei InSb wurde ein doppelter Nulldurchgang des HaLl-Koeffizienten beobachtet, wie Abb. 5 zeigt. Es handelt sich bei den drei dargestellten Präparaten um polykristallines Material, so daß nähere Angaben nicht möglich sind.

Fassen wir die hier mitgeteilten Ergebnisse kurz zusammen, so können wir feststellen: Die Potentialmessungen zeigen, daß die in allen Fällen vorhandene positive HaLL-Spannung des Volumens bei InAs erhalten bleibt. Die zwischen den Hall-Sonden gemessene HaLL-Spannung setzt sich aus einem positiven Volumenanteil und den negativen Oberflächenanteilen zusammen. Die in der Oberfläche erzeugte negative HaLl-Spannung ist größer als die positive Spannung des Volumens, so daß man schließlich eine negative Gesamtspannung mißt. Unter Zuhilfenahme von experimentellen Daten lassen sich einige Abschätzungen über HALL-Koeffizient und Beweglichkeit in den Oberflächenschichten vornehmen.

Für die Überlassung der Präparate haben wir den Herren Dr. O. G. Folberth und Dr. H. A. Schell vielmals zu danken.

\title{
Zur Theorie der Wärmeleitung in Isolatoren* I
}

\author{
Von Harry Paul \\ Aus dem Theoretisch-Physikalischen Institut der Universität Jena \\ (Z. Naturforschg. 14 a, 535-540 [1959]; eingegangen am 6. Dezember 1958)
}

\begin{abstract}
In die Beschreibung der Wärmeleitung werden - im Sinne des von Lebowitz und Bergmann vorgeschlagenen „New Approach to Non-equilibrium Processes“ - die Wärmebäder, die experimentell den Ablauf des Wärmeleitungsvorganges erzwingen, mit eingeschlossen. Es ergeben sich Randbedingungen für die mittleren Phononenbesetzungszahlen auf den Begrenzungen des Kristalls, die sich mit den Wärmebädern in Kontakt befinden. Bei hohen Temperaturen erhält man denselben Wert für die Wärmeleitfähigkeit wie in der Peiercsschen Theorie.
\end{abstract}

Will man die Wärmeleitung in einem Isolator vom quantenmechanischen Standpunkt aus studieren, so steht einem die von Peierls ${ }^{1}$ entwickelte Theorie zur Verfügung, die auf der Phononenkonzeption basiert. Das Phononengas ist im Fall stationärer Prozesse der Boltzmannschen Stoßgleichung unterworfen. Die Aufgabe besteht darin, solche Lösungen dieser Gleichung aufzusuchen, die es erlauben, jedem Raumpunkt - zumindest genähert eine Temperatur zuzuordnen. Der betrachtete Kristall wird dabei als unendlich ausgedehnt angesehen. Um jedoch bei sehr tiefen Temperaturen mit dem Experiment in Übereinstimmung zu bleiben, hat man nach CASImir ${ }^{2}$ einen ganz anderen Weg einzuschlagen: Man hat einen Kristall zu betrachten, der zumindest in einer Dimension nur endlich ausgedehnt ist, und sich vorzustellen, daß die Randpunkte verschiedene Temperaturen besitzen und entsprechend der jeweiligen Temperatur Phononen emittieren. (Von Casimir wurde der Fall eines unendlich langen

\footnotetext{
* Gekürzte Fassung der Jenaer Dissertation 1958.

1 R. Peierls, Ann. Phys., Lpz. 3, 1055 [1929].

2 H. G. B. Casimir, Physica 5, 495 [1938].
}

Zylinders durchgerechnet; dort hat man einen genähert linearen Temperaturverlauf längs des Zylinders anzunehmen.)

In der vorliegenden Arbeit wird nun versucht, diese beiden scheinbar so völlig verschiedenen Behandlungsmethoden miteinander in Einklang zu bringen. Das geschieht durch Einbeziehung der Wärmebäder in die Betrachtung entsprechend den von Lebowitz und Bergmann ${ }^{3,4}$ entwickelten Ideen. Ein Wärmebad wird durch eine kanonische Gesamtheit repräsentiert; ein Mitglied dieser Gesamtheit tritt höchstens einmal (und auch dann nur kurzzeitig) in Wechselwirkung mit einem System aus der Gesamtheit, die den betrachteten (endlich ausgedehnten) Kristall wiedergibt. Wenn man zum Aufbau der kanonischen Gesamtheiten für die Wärmebäder speziell Exemplare des Kristalls benutzt, kann man die Wechselwirkung leicht übersehen. Der Einfluß der Wärmebäder äußert sich in Randbedingungen für die mittleren Phononenbesetzungszahlen.

3 P. G. Bergmann u. J. L. Lebowitz, Phys. Rev. 99, 578 [1955].

4 J. L. Lebowitz u. P. G. Bergmann, Ann. Physics 1, 1 [1957]. 


\section{$\S$ 1. Das Phononengas}

Es werde ein Kristall betrachtet, der durch die Gittervektoren

$$
\mathfrak{g}=\sum_{i=1}^{3} n_{i} \mathfrak{a}^{(i)} \quad\left[n_{i}(i=1,2,3) \text { ganz }\right]
$$

gekennzeichnet sei. (Wir beschränken uns der Einfachheit halber auf primitive Gitter.) $\Re$ sei ein beliebiger Vektor des reziproken Gitters. $\mathfrak{S}^{\mathfrak{g}}$ symbolisiere die Verrückung des Gitterpunktes g aus der Ruhelage.

Für die potentielle Energie des Gitters schreiben wir

$$
\begin{aligned}
& \Phi=\Phi_{0}+\frac{1}{2 !} \sum_{\substack{g \\
k k^{\prime}}} \Phi_{k k^{\prime}}^{g} g_{k}^{\prime}, s_{k}^{g} s_{k^{\prime}}^{q^{\prime}}
\end{aligned}
$$

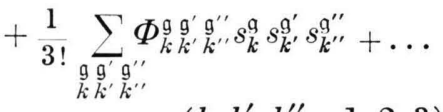

$$
\begin{aligned}
& \left(k, k^{\prime}, k^{\prime \prime}=1,2,3\right) .
\end{aligned}
$$

$N$ sei die Zahl der Gitterpunkte im betrachteten Volumen $V$.

Die $3 N$ Normalschwingungen des Gitters wählen wir in der Form linear polarisierter fortlaufender ebener Wellen, die durch einen Wellenzahlvektor und einen Zeiger $s(s=1,2,3)$, der die Polarisation angibt, charakterisiert werden. (Zu einem Vektor gehören drei verschiedene Polarisationsrichtungen, die wir durch die zueinander senkrechten Einheitsvektoren $\mathfrak{e}^{s}(\mathfrak{f}) \quad[s=1,2,3] \quad$ kennzeichnen.) Die
Kreisfrequenz $\omega$ ergibt sich als eine durch die Gitterstruktur festgelegte Funktion von $\mathfrak{f}, s$ :

$$
\omega=\omega_{s}^{t} \text {. }
$$

Durch die Quantisierung gelangt man zu der Vorstellung des Phononengases (vgl. z. B. Leibrried ${ }^{5}$ ) :

Durch $f, s$ zu charakterisierende Quasiteilchen (Phononen) fliegen mit der Geschwindigkeit

$$
\mathfrak{v}_{s}^{\ddagger}=\operatorname{grad}_{\mathfrak{f}} \omega_{s}^{\ddagger}
$$

umher. Die Energiestromdichte ist durch

$$
\mathfrak{q}=\frac{1}{V} \sum_{\mathfrak{i} s} \hbar \omega_{s}^{\mathfrak{t}} N_{s}^{\mathfrak{t}} \mathfrak{v}_{s}^{\mathfrak{q}}
$$

gegeben; dabei sind die $N_{s}^{\sharp}$ die mittleren Besetzungszahlen der Phononen.

Falls thermisches Gleichgewicht (Temperatur $T$ ) vorliegt, gilt

$$
N_{s}^{\ddagger}=N_{s}^{\ddagger}\{T\} \equiv\left(\exp \left\{\hbar \omega_{s}^{\ddagger} / k T\right\}-1\right)^{-1}
$$

\section{( $k$ Boltzmann-Konstante).}

Die mittleren Besetzungszahlen an der Stelle $r$ ändern sich durch zwei Prozesse, nämlich durch Konvektion

$$
\left.\dot{N}_{s}^{\mathfrak{f}}\right|_{\text {Konvektion }}=-\mathfrak{b}_{s}^{\mathfrak{f}} \operatorname{grad}_{\mathrm{r}} N_{s}^{\mathfrak{t}}(\mathfrak{r})
$$

und durch Stöße zwischen den Phononen.

$\left.\dot{N}_{s}^{\mathfrak{f}}\right|_{\text {Sto } B}$ hat, wenn nur die von den kubischen Termen in (1) herrührenden Stöße berücksichtigt wer. den, den Wert (Formel [92.11] bei Leibfried ${ }^{5}$ )

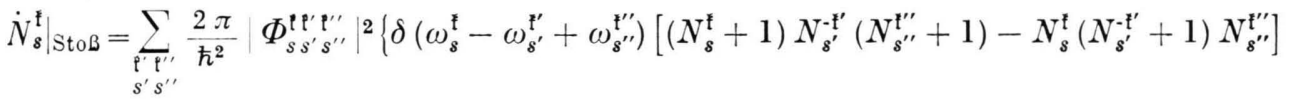

$$
\begin{aligned}
& +\delta\left(\omega_{s}^{\mathrm{f}}+\omega_{s^{\prime}}^{\mathrm{f}^{\prime}}-\omega_{s^{\prime \prime}}^{\mathrm{f}^{\prime \prime \prime}}\right)\left[\left(N_{s}^{\mathrm{t}}+1\right)\left(N_{s^{\prime}}^{\mathrm{t}^{\prime}}+1\right) N_{s^{\prime \prime}}^{\mathrm{t} \mathrm{t}^{\prime \prime}}-N_{s}^{\mathrm{t}} N_{s^{\prime}}^{\mathrm{t}^{\prime}}\left(N_{s^{\prime \prime}}^{-\mathrm{f}^{\prime \prime}}+1\right)\right] \\
& \left.+\delta\left(\omega_{s}^{\ddagger}-\omega_{s^{\prime}}^{\mathrm{f}^{\prime}}-\omega_{s^{\prime \prime}}^{\mathrm{f}^{\prime \prime}}\right)\left[\left(N_{s}^{\mathrm{\ddagger}}+1\right) N_{s^{\prime}}^{-\mathrm{f}^{\prime}} N_{s^{\prime \prime}}^{-\mathrm{f}^{\prime \prime}}-N_{s}^{\ddagger}\left(N_{s^{\prime}}^{-\mathrm{t}^{\prime}}+1\right)\left(N_{s^{\prime \prime}}^{-\mathrm{f}^{\prime \prime}}+1\right)\right]\right\} \text {. }
\end{aligned}
$$

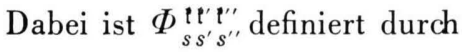

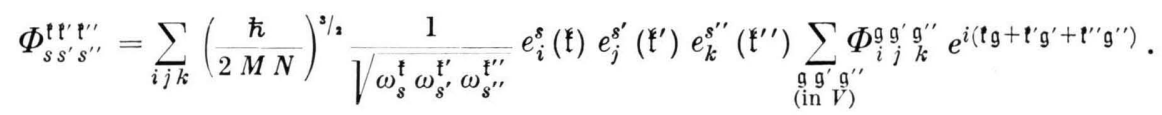

( $N$ ist die Zahl der Atome in $V, M$ die Masse eines Atoms, $e_{i}^{\delta}(\mathfrak{f})$ die $i$-Komponente des Einheitsvektors $\mathrm{e}^{s}(\mathfrak{f})$ usf.)

Es läßt sich leicht zeigen, daß $\Phi_{s s^{\prime} s^{\prime \prime}}^{\mathrm{tP}^{\prime} \mathrm{p}^{\prime \prime}}$ den Faktor

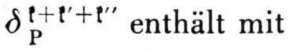

$$
\delta_{\mathrm{P}}^{\mathfrak{l}+\mathfrak{l}^{\prime}+\mathfrak{l}^{\prime \prime}}= \begin{cases}1 & \text { für } \\ 0 & \text { sonst. }\end{cases}
$$

Für den stationären Fall muß

$$
\dot{N}_{s \mid \text { Konvektion }}^{\mathrm{f}}+\left.\dot{N}_{s}^{\mathrm{f}}\right|_{\text {Sto } B}=0
$$

sein (Boltzmannsche Stoßgleichung).

5 G. Letbrried, Handbuch d. Physik VII/1, S. 290, Berlin 1955. 


\section{§ 2. Wechselwirkung mit äußeren Wärmebädern}

Das betrachtete System werde durch eine Gesamtheit S repräsentiert, ein Reservoir durch eine Gesamtheit R. Bergmann und Lebowitz entwickelten die folgenden grundsätzlichen Vorstellungen über den Wechselwirkungsmechanismus zwischen $\mathrm{S}$ und $\mathrm{R}$ :

Ein Element $\mathrm{s}$ aus $\mathrm{S}$ tritt kurzzeitig mit einem Element $\mathrm{r}$ aus $\mathrm{R}$ in Wechselwirkung (,impulsive interaction"), im nächsten Zeitpunkt mit einem anderen Element $\mathrm{r}^{\prime}$ aus $\mathrm{R}$ usf. Um eine Rückwirkung des Systems auf das Reservoir auszuschließen, wird die Annahme gemacht, daß jedes Element aus $R$ im Laufe der Zeit höchstens einmal in Aktion tritt. Das Reservoir bleibt somit zeitlich unverändert, ist unerschöpflich in seiner Energie und besitzt selbst keine inneren Gradienten (z. B. keinen Temperaturgradienten). Dem Reservoir sind definierte Werte thermodynamischer Größen zuzuschreiben (z. B. ein Temperaturwert), während den Punkten im Innern des Systems i. a. keine solchen Werte zuzuordnen sind.

Es wird angenommen, daß $\mathrm{s}$ und $\mathbf{r}$ vor dem Einsetzen der Wechselwirkung (vor dem „Stoß“) statistisch unabhängig sind; dann kann man also über $\mathrm{S}$ und $\mathrm{R}$ unabhängig mitteln. Die Mittelung ergibt die Beschreibung der zeitlichen Änderung des Systems infolge der Wechselwirkung mit einem Reservoir.

Wir wenden die geschilderten Ideen auf die Theorie der Wärmeleitung an. Zum Aufbau von R benutzen wir geschickterweise Exemplare eines Kristalls, für den die Funktion $\omega_{s}^{\ddagger}$ mit der unseres betrachteten Systems übereinstimmt, mit anderen Worten der dieselben Phononensorten besitzt wie unser System. Wir bemerken, daß ein solcher Kristall keineswegs ein Isolator zu sein braucht; die Existenz eines Elektronengases und die Möglichkeit von Stößen zwischen Elektronen und Phononen stören nicht.

$\mathrm{R}$ repräsentiere ein $\mathrm{Wärmebad}$ der Temperatur $T_{1}$; wir haben also $\mathrm{R}$ als eine kanonische Gesamtheit zu wählen. Die Gesamtheit $\mathrm{S}$ ist durch die mittleren Besetzungszahlen $N_{s}^{\ddagger}$ charakterisiert.

Wir studieren die Wechselwirkung zwischen s und r : Die Trennwand zwischen Wärmebad und System liege in der $y-z$-Ebene. Die positive $x$-Richtung zeige in das System hinein. Die Phononen mit $v_{s}^{\mathfrak{f}}>0 \quad\left(v_{s}^{\mathfrak{f}}=x\right.$-Komponente von $\left.\mathfrak{v}_{s}^{\mathfrak{f}}\right)$ aus $\mathrm{r}$ fliegen dann einfach ungestört durch die Grenzfläche hin- durch in $\mathrm{s}$ hinein; s seinerseits verliert Phononen an $\mathrm{r}$ mit $v_{s}^{\ddagger}<0$. Wenn wir über $\mathrm{R}$ und $\mathrm{S}$ mitteln, kommen wir zu dem Ergebnis: Während $\mathrm{d} t$ treten im Mittel aus dem Wärmebad

$$
\frac{1}{V} N_{s}^{\ddagger}\left\{T_{1}\right\} v_{s}^{\ddagger} \mathrm{d} t \quad\left(v_{s}^{\ddagger}>0\right)
$$

Phononen der Sorte $\mathfrak{f}, s$ (mit $v_{s}^{\ddagger}>0$ ) durch die Flächeneinheit in das System, unabhängig davon, was im Reservoir an Stößen (evtl. auch mit Elektronen) geschieht. (Übrigens findet im Mittel dort in Wahrheit keine Änderung der Besetzungszahlen durch Stöße statt, da thermisches Gleichgewicht vorliegt.) Im System selbst haben wir überall dort einen von Null verschiedenen Wert von $\left.\dot{N}_{s}^{\ddagger}\right|_{\text {Stoß }}$, wo die $N_{s}^{\ddagger}$ keine thermische Verteilung repräsentieren.

Es liege nun ein stationärer Zustand vor. Damit ein Wärmestrom fließen kann, muß natürlich noch mindestens ein zusätzliches Wärmebad mit einer Temperatur $T_{2} \neq T_{1}$ an das System gekoppelt sein. Wir interessieren uns dafür, was an der Grenzfläche zwischen dem System und dem ersten Reservoir (Temperatur $T_{1}$ ) geschieht. Es werde eine flache zylindrische Dose ins Auge gefaßt. Die eine Deckfläche der Größe Uf liege in der Trennwand $x=0$ um den Punkt $x=0, y, z$; die andere innerhalb des Systems in der Fläche $x=\varepsilon$. Wir betrachten nur Phononen der Sorte $\mathfrak{f}, s$ mit $v_{s}^{\ddagger}>0$. In der Zeit $\mathrm{d} t$ fliegen offenbar

$$
\frac{1}{V} N_{s}^{\ddagger}\left\{T_{1}\right\} v_{s}^{\ddagger} \Delta f \mathrm{~d} t
$$

Phononen durch die eine Deckfläche aus dem Reservoir in die Dose hinein, während durch die andere Deckfläche

$$
\frac{1}{V} N_{s}^{\ddagger}(\varepsilon, y, z) v_{s}^{\mathfrak{f}} \Delta f \mathrm{~d} t
$$

Phononen aus der Dose austreten.

Die Zahl der Phononen, die durch die restliche Fläche strömen, ist offenbar von der Ordnung $\varepsilon(\Delta f)^{1 / 2} \mathrm{~d} t$. Während $\mathrm{d} t$ werden im Volumen $V$ $\left.\dot{N}_{8}^{t}\right|_{\text {Sto } B} \cdot \mathrm{d} t$ Phononen erzeugt, also in der Dose

$$
\left.\dot{N}_{s}^{\ddagger}\right|_{\text {Sto } B} \frac{\varepsilon \Delta f}{V} \mathrm{~d} t .
$$

Dabei ist $\dot{N}_{s}^{\mathrm{f}}||_{\text {Sto } \mathrm{B}}$ der Ausdruck (1.6).

Insgesamt soll die Zahl der Phononen in der Dose zeitlich konstant bleiben (Forderung der Stationarität). Wenn wir den Grenzübergang $\varepsilon \rightarrow 0, \Delta f \rightarrow 0$ 
so vornehmen, daß $\varepsilon(\Delta f)^{-1 / 2}$ ebenfalls gegen Null geht (flache Dose), so folgt daraus

$$
\lim _{\varepsilon \rightarrow 0} N_{s}^{\ddagger}(\varepsilon, y, z)=N_{s}^{\mathfrak{\ddagger}}\left\{T_{1}\right\} \quad \text { für } \quad v_{s}^{\ddagger}>0 .
$$

Das eindimensionale Wärmeleitungsproblem (bei $x=0$ werde die Temperatur $T_{1}$, bei $x=L$ die Temperatur $T_{2}$ aufrechterhalten) ist somit in folgender Weise zu behandeln:

Es ist die Boltzmannsche Stoßgleichung (1.9) zu lösen unter der Randbedingung

$$
\begin{array}{lll}
N_{s}^{\ddagger}(0)=N_{s}^{\ddagger}\left\{T_{1}\right\} & \text { für } & v_{s}^{\ddagger}>0, \\
N_{s}^{\ddagger}(L)=N_{s}^{\ddagger}\left\{T_{2}\right\} & \text { für } & v_{s}^{\ddagger}<0 .
\end{array}
$$

Die vorstehende Forderung läßt sich in der Tat befriedigen: Es handëlt sich um ein System von $3 N$ gewöhnlichen Differentialgleichungen 1. Ordnung für $3 N$ Funktionen; wir haben also $3 N$ Integrations- konstanten und offenbar genau so viele Bedingungsgleichungen (3).

Es soll nun untersucht werden, wie weit das neue Verfahren für hohe Temperaturen von der Peierlsschen Behandlungsmethode abweicht.

\section{§ 3. Wärmeleitung bei hohen Temperaturen}

Wir beschränken uns auf das eindimensionale Problem: Alle interessierenden Größen sollen nur von $x$ abhängen.

Im späteren Beweisgang werden wir wesentlich von der Tatsache Gebrauch machen, daß die Wärmestromdichte $q$, die einer stationären Phononenverteilung entspricht, unabhängig von $x$ ist. Diesen physikalisch sinnvollen Sachverhalt wollen wir zunächst beweisen. $\left.\dot{N}_{s}^{\ddagger}\right|_{\text {Stoß }}$ läßt sich, wie man leicht nachprüft, in der folgenden Form schreiben:

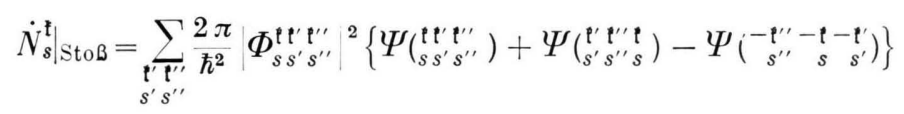

mit

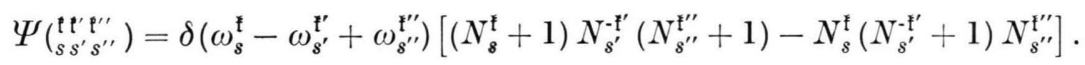

Wenn man die Stoßgleichung $v_{s}^{\ddagger}\left(\partial N_{s}^{\ddagger} / \partial x\right)=\left.\dot{N}_{s}^{\ddagger}\right|_{\text {Stoß }}$ mit $\hbar \omega_{s}^{\ddagger} V^{-1}$ multipliziert und über $\mathfrak{f}, s$ summiert, entsteht auf der linken Seite

$$
\frac{\partial}{\partial x} \frac{1}{V} \sum_{i s} \hbar \omega_{s}^{\ddagger} v_{s}^{\ddagger} N_{s}^{\ddagger}=\frac{\partial q}{\partial x} .
$$

Rechter Hand hat man $\left.\frac{1}{V} \sum_{\uparrow s} \hbar \omega_{s}^{\ddagger} \dot{N}_{s}^{\ddagger}\right|_{\text {Sto }}$, was nach (1) gleich

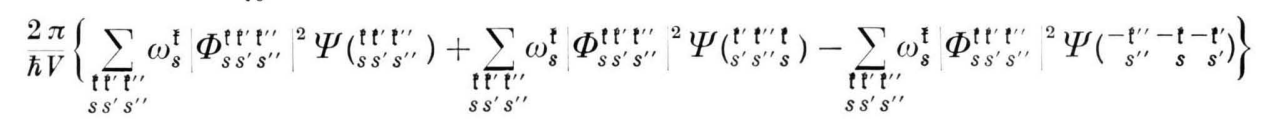

ist.

Durch Umbenennung der Summationsindizes in der zweiten und dritten Summe kann man erreichen, daß das Argument von $\Psi$ stets ${ }^{\prime \ell^{\prime} s^{\prime} s^{\prime \prime}} s^{\prime \prime}$ lautet. Wenn man beachtet, daß $\Phi_{s s^{\prime} s^{\prime \prime}}^{\mathrm{P}^{\prime} \mathrm{p}^{\prime \prime}}$ gegen die Vertauschung zweier Indexpaare (z. B. $\left.\mathfrak{f} s \longleftrightarrow \mathfrak{f}^{\prime} s^{\prime}\right)$ invariant ist ${ }^{5}$, und daß außerdem die Relationen ${ }^{5}$

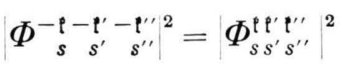

$$
\begin{aligned}
& \omega_{s}^{-\mathfrak{t}}=\omega_{s}^{\mathfrak{t}}
\end{aligned}
$$

gelten, so erhält man leicht für (4) den Ausdruck

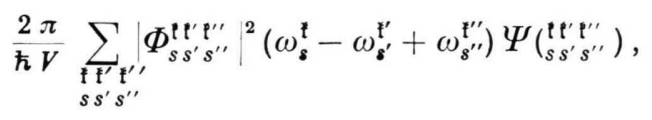

der wegen des in $\Psi$ steckenden Faktors

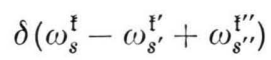

verschwindet. Also ist in der Tat $\partial q / \partial x=0$.

Nun vergleichen wir zwei Lösungen der Stoßgleichung, nämlich eine nach dem Peierlsschen Ansatz konstruierte Lösung $N_{s}^{\ddagger}(x)$ und eine nach unserem Verfahren gewonnene Lösung $M_{s}^{\mathfrak{f}}(x)$ miteinander. Peierls nimmt an, daß man jeder Stelle $x$ genähert eine Temperatur zuordnen kann:

$$
N_{s}^{\ddagger}(x)=N_{s}^{\ddagger}\{T(x)\}+n_{s}^{\ddagger}(x) .
$$

( $n_{s}^{\ddagger}$ beschreibt eine kleine Abweichung vom thermodynamischen Gleichgewicht.) Randbedingungen werden nicht gestellt. Offenbar genügt, wenn wir $T(0)$ 
mit $T_{1}$ und $T(L)$ mit $T_{2}$ bezeichnen, $N_{s}^{\ddagger}(x)$ den Randbedingungen

$$
\begin{aligned}
& N_{s}^{\ddagger}(0)=N_{s}^{\ddagger}\left\{T_{1}\right\}+n_{s}^{\ddagger}(0) \\
& N_{s}^{\ddagger}(L)=N_{s}^{\ddagger}\left\{T_{2}\right\}+n_{s}^{\ddagger}(L) \quad \text { für } \quad v_{s}^{\ddagger}>0, \\
& v_{s}^{\ddagger}<0 .
\end{aligned}
$$

(Diese Gleichungen gelten für alle $\mathfrak{f}, s$; also im bes. für die angeschriebenen Werte.) $M_{s}^{\ddagger}(x)$ dagegen soll die Randbedingungen

$$
\begin{array}{llc}
M_{s}^{\ddagger}(0)=N_{s}^{\ddagger}\left\{T_{1}\right\} & \text { für } \quad v_{s}^{\ddagger}>0, \\
M_{s}^{\ddagger}(L)=N_{s}^{\ddagger}\left\{T_{2}\right\} & \text { für } \quad v_{s}^{\ddagger}<0
\end{array}
$$

erfüllen.

Die rechten Seiten der beiden Randbedingungen unterscheiden sich nur durch den Summanden $n_{s}^{\ddagger}(0)$ bzw. $n_{s}^{\ddagger}(L)$. Wir wollen uns von nun an auf den Fall hoher Temperaturen beschränken. Wir bezeichnen mit $T$ den Mittelwert $\left(T_{1}+T_{2}\right) / 2$; dann sind $N_{s}^{\ddagger}\left\{T_{1}\right\}$ und $N_{s}^{\ddagger}\left\{T_{2}\right\}$ von der Ordnung $T$ (wir setzen voraus, daß die Ordnung von $T_{2}-T_{1}$ höchstens gleich Eins ist) $n_{s}^{\ddagger}$ ist von der Ordnung $T^{-1}$ (vgl. LEIBFried ${ }^{5}$ ). Man wird annehmen können, daß auch $N_{s}^{\ddagger}(x)-M_{s}^{\ddagger}(x)$ von der Ordnung $T^{-1}$ ist. Wir schreiben

$$
\begin{aligned}
M_{s}^{\ddagger}(x) & =N_{s}^{\ddagger}(x)+h_{s}^{\ddagger}(x), \\
h_{s}^{\ddagger}(x) & =O\left(T^{-1}\right) .
\end{aligned}
$$

$h_{s}^{\ddagger} \mathrm{mu} \beta$ dann offenbar den Randbedingungen

$$
\begin{array}{lll}
h_{s}^{\ddagger}(0)=-n_{s}^{\ddagger}(0) & \text { für } \quad v_{s}^{\ddagger}>0, \\
h_{s}^{\ddagger}(L)=-n_{s}^{\ddagger}(L) & \text { für } \quad v_{s}^{\ddagger}<0
\end{array}
$$

genügen.

Nach Voraussetzung sind $N_{s}^{\ddagger}(x)$ und $M_{s}^{\ddagger}(x)$ Lösungen der Stoßgleichung; schreibt man die Stoßgleichung für $N_{s}^{\ddagger}$ und $M_{s}^{\ddagger}$ auf, so ergibt sich durch Subtraktion

mit

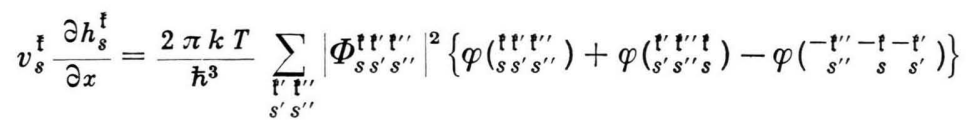

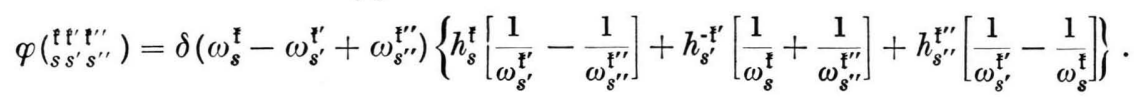

Dabei wurden auf der rechten Seite von (11) die Terme von erster und höherer Ordnung in $T^{-1}$ gegen 1 vernachlässigt. Es wurde außerdem berücksichtigt, daß $T_{2}-T_{1}$ von der Ordnung 1 ist; daher konnte $N_{s}^{\ddagger}\{T(x)\}$ durch $N_{s}^{\ddagger}\{T\} \approx k T / \hbar \omega_{s}^{\ddagger}$ ersetzt werden.

Statt (11) schreiben wir abgekürzt

$$
v_{s}^{\ddagger} \frac{\partial h_{s}^{\mathfrak{t}}}{\partial x}=\sum_{\mathbb{\ell}^{\prime} s^{\prime}} c_{s s^{\prime}}^{\mathfrak{p} \mathfrak{p}^{\prime}} h_{s^{\prime}}^{\mathfrak{f}^{\prime}} .
$$

Wir erwähnen, daß die rechte Seite dieser Gleichung bei einem ganz anderen Problem ebenfalls in Erscheinung tritt. Stellt man sich nämlich die Aufgabe, die zeitliche Änderung einer ortsunabhängigen Verteilung

$$
\begin{aligned}
N_{s}^{\ddagger} & =N_{s}^{\ddagger}\{T\}+g_{s}^{\ddagger}, \\
g_{s}^{\ddagger} & =O\left(T^{-1}\right)
\end{aligned}
$$

$\mathrm{zu}$ berechnen, so liefert die Rechnung in der oben angegebenen Näherung

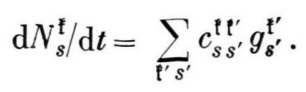

Die Lösung von (12) erfolgt (vgl. etwa Anm. ${ }^{6}$ ) durch den Ansatz

$$
h_{s}^{\ddagger}=a_{s}^{\ddagger} e^{\lambda x} .
$$

$\lambda$ und die $a_{s}^{\ddagger}$ bestimmen sich aus der Gleichung

$$
D \mathfrak{a}=\lambda \mathfrak{a} .
$$

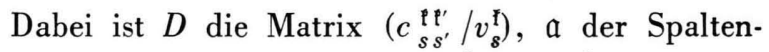
vektor mit den Komponenten $a_{s}^{\ddagger}$. Die $a_{s}^{\ddagger}$, die ja nur bis auf einen gemeinsamen Faktor fixiert sind, können offenbar als $T$-unabhängige Zahlen gewählt werden.

Man hat also die mathematische Aufgabe, die Matrix $D$ zu diagonalisieren, vor sich.

Sei $\lambda \neq 0$ ein $p$-fach entarteter Eigenwert von $D$. Dann gibt $\lambda$ Anlaß zu $p+1$ Lösungen vom Typ

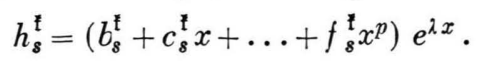

Bezeichnen wir die $x$-Komponente der Stromdichte, die zur Verteilung $N_{s}^{\ddagger}$ gehört, mit $q\left\{N_{s}^{\ddagger}\right\}$ :

$$
q\left\{N_{s}^{\ddagger}\right\}=\frac{1}{V} \sum_{\mathfrak{i} s} \hbar \omega_{s}^{\ddagger} v_{s}^{\ddagger} N_{s}^{\ddagger}
$$

$\left(q\left\{N_{s}^{\ddagger}\right\}\right.$ ist eine lineare Bildung in $\left.N_{s}^{\ddagger}\right)$, so können 
wir sagen: da $N_{s}^{\ddagger}$ und $M_{s}^{\ddagger}=N_{s}^{\ddagger}+h_{s}^{\ddagger}$ Lösungen der Stoßgleichung sind, müssen $q\left\{N_{s}^{\ddagger}\right\}$ und $q\left\{N_{s}^{\ddagger}+h_{s}^{\ddagger}\right\}$ von $x$ unabhängig sein; dann darf aber auch $q\left\{h_{s}^{\ddagger}\right\}=q\left\{N_{s}^{\ddagger}+h_{s}^{\ddagger}\right\}-q\left\{N_{s}^{\ddagger}\right\}$ nicht von $x$ abhängen.

Setzen wir für $h_{s}^{\ddagger}$ den Wert (17) ein, so erhalten wir für $q\left\{h_{s}^{\mathrm{f}}\right\}$ einen Ausdruck der Form

$$
\left(q_{0}+q_{1} x+\ldots+q_{p} x^{p}\right) e^{\lambda x},
$$

der offenbar nur dann $x$-unabhängig ist, wenn alle Koeffizienten verschwinden, woraus $q\left\{h_{s}^{\ddagger}\right\}=0$ folgt.

Falls $\lambda=0$ ist, kann man nicht so schließen. In diesem Fall ist ein Eigenvektor a der Matrix $D$ zugleich ein Eigenvektor der Matrix $C=\left(c_{s s^{\prime}}^{\mathfrak{\ddagger} \mathfrak{f}^{\prime}}\right)$ (zum Eigenwert 0), wie man sofort sieht. Die Gl. (14) sagt dann aus: die Verteilung $N_{s}^{\ddagger}=N_{s}^{\ddagger}\{T\}+a_{s}^{\ddagger} T^{-1}$ ist (genähert) stationär. Nun darf aber, wenn der Stoßmechanismus physikalisch vernünftig ist, nur eine thermische Verteilung stationär sein. (Der Stoßmechanismus muß jede Verteilung, die nicht dem thermischen Gleichgewicht entspricht, im Laufe der Zeit in ein solches Gleichgewicht überführen!) Es muß also

$$
N_{s}^{\ddagger}\{T\}+a_{s}^{\ddagger} T^{-1}=N_{s}^{\ddagger}\{T+\Delta T\}
$$

sein; daraus folgt

$$
a_{s}^{\mathfrak{t}}=\mathrm{const} / \omega_{s}^{\mathrm{t}} \text {. }
$$

Der Eigenvektor $\mathfrak{a}$ ist somit - bis auf einen Normierungsfaktor - eindeutig bestimmt, also ist der Eigenwert $\lambda=0$ der Matrix $D$ nicht entartet.

$\mathrm{Da} \omega_{s}^{\ddagger}$ in $\mathfrak{f}$ symmetrisch ist, folgt aus (19)

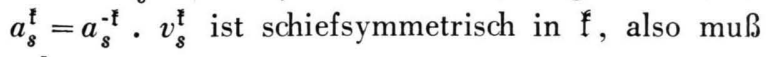
gelten

$$
q\left\{a_{s}^{\ddagger}\right\}=0 .
$$

Wir können somit sagen: Für jede spezielle Lösung $h_{s}^{\ddagger}$ des Systems (11) verschwindet $q\left\{h_{s}^{\ddagger}\right\}$; also gilt dasselbe auch für die Überlagerung der speziellen Lösungen, die den Randbedingungen (10) genügt. Nach (9) ist

$$
q\left\{M_{s}^{\ddagger}\right\}=q\left\{N_{s}^{\ddagger}\right\}+q\left\{h_{s}^{\ddagger}\right\}
$$

und nach dem Gesagten offenbar

$$
q\left\{M_{s}^{\ddagger}\right\}=q\left\{N_{s}^{\ddagger}\right\} .
$$

Die Berechnung des Stromes liefert also (im Rahmen der benutzten Näherung) in unserer Theorie dasselbe Ergebnis wie in der Peierlsschen Theorie. Das gleiche gilt dann offenbar auch für die Berechnung der Wärmeleitfähigkeit.

Im Teil II dieser Arbeit wird das Leitfähigkeitsproblem bei sehr tiefen Temperaturen behandelt.

6 E. Kамке, Differentialgleichungen - Lösungsmethoden und Lösungen I, Geest \& Portig, Leipzig 1956.

\title{
Zur Theorie der Wärmeleitung in Isolatoren* II
}

\author{
Von Harry PaUl \\ Aus dem Theoretisch-Physikalischen Institut der Universität Jena \\ (Z. Naturforschg. 14 a, 540—547 [1959]; eingegangen am 6. Dezember 1958)
}

\begin{abstract}
Die vom Verf. früher entwickelte Theorie der Wärmeleitung wird für sehr tiefe Temperaturen diskutiert. Man gelangt für den Fall eines langen Zylinders zu einer Integralgleichung, die näherungsweise gelöst wird. Das Resultat stimmt mit dem 1938 von Casımir gegebenen überein; allerdings kann - im Gegensatz zu Casimir - nur den Punkten des Zylinders eine Temperatur zugeschrieben werden, die sich in Kontakt mit den Wärmebädern befinden. Für eine flache Dose (Dicke $D$ ) ist für $T \rightarrow 0$ die Wärmeleitfähigkeit $\lambda$ proportional zu $D T^{3}$, wenn man von den Stößen der Phononen untereinander absieht. Bei Berücksichtigung der Stöße in 1 . Näherung wird $\lambda$ um einen $D^{2} T^{8}$ proportionalen Term vermindert.
\end{abstract}

Bekanntlich divergiert in der PeierLsschen Theorie der Wärmeleitung für Isolatoren die Wärmeleitfähigkeit $\lambda$, wenn die Anharmonizität des Gitterpotentials verschwindet. Wie ist die unendlich große Wärmeleitfähigkeit in Wahrheit zu verstehen?

Wir weisen darauf hin, daß die Peierlssche Rech-

- Gekürzte Fassung der Jenaer Dissertation 1958.

1 G. Leibfried, Handb. d. Physik VII/1, 290 [1955]. nung (vgl. etwa Leibfried ${ }^{1}$ ) auf der ausdrücklichen Voraussetzung

$$
\left|n_{s}^{\ddagger}(\mathfrak{r})\right| \ll N_{s}^{\ddagger}\{T(\mathrm{r})\}
$$

beruht. $\left[n_{s}^{\sharp}(\mathfrak{r})\right.$ beschreibt die Abweichung von der thermischen Verteilung nach $(I, 3.6)^{* *}$.]

** Mit I versehene Formelnummern beziehen sich auf den Teil I dieser Arbeit ${ }^{2}$. 